\title{
X-ray absorption in Compton-thin AGN: the predictions of a model revisited
}

\author{
A. Lamastra, G. C. Perola, and G. Matt
}

\begin{abstract}
Dipartimento di Fisica "E. Amaldi", Università degli Studi Roma Tre, via della Vasca Navale 84, 00146 Roma, Italy
\end{abstract} e-mail: lamastra@fis.uniroma3.it

Received 28 November 2007 / Accepted 24 April 2008

\begin{abstract}
Context. The evidence of a decrease with increasing luminosity in the fraction $f_{\text {abs }}$ of absorbed and Compton-thin among X-rayselected $(2-10 \mathrm{keV})$ AGN is observationally well supported, while that of an increase in $f_{\text {abs }}$ with redshift is fairly controversial. In a previous paper, the gravitational effect of the SMBH on the molecular interstellar gas, in the central region of the host galaxy, was shown to predict an anti-correlation between $f_{\mathrm{abs}}$ and the black-hole mass $M_{\mathrm{BH}}$.

Aims. The most recent findings on the distribution of the Eddington ratio $\lambda=L_{\mathrm{b}} / L_{\mathrm{E}}$ as a function of $M_{\mathrm{BH}}$ and $z$ are used to convert that relationship into one between $f_{\mathrm{abs}}$ and both bolometric $\left(L_{\mathrm{b}}\right)$ and X-ray $\left(L_{\mathrm{X}}\right)$ luminosities at various values of $z$.

Methods. The findings for $\lambda\left(M_{\mathrm{BH}}, z\right)$ are properly treated to ensure completeness in the prediction of $f_{\mathrm{abs}}$ above a certain luminosity, at values of $z=0.1,0.35,0.7$, and $\geq 1$. To verify the consequence of these findings alone, we first adopted a distribution of gas surface density $\Sigma$, observed in a sample of local spiral galaxies, irrespective of the galaxy morphological type and $z$.

Results. Assuming the Eddington limit, $\lambda=1$, in the $\lambda\left(M_{\mathrm{BH}}, z\right)$ distribution as a "natural" cut-off, the predictions are consistent with the existence of an anti-correlation between $f_{\text {abs }}$ and $L_{\mathrm{X}}$, but they fail to reproduce an increase in $f_{\text {abs }}$ with $z$. Because the early type galaxies are on average much poorer in molecular gas than late type ones, a quantitative agreement with the local value of $f_{\text {abs }}$ requires the existence of a correlation between $\Sigma$ and the central activity. An increase in typical values of $\Sigma$ with $z$, correlated with the activity, might explain an increase in $f_{\text {abs }}$ with $z$. However, $f_{\text {abs }}$ could hardly exceed about 0.3 at the highest luminosities.
\end{abstract}

Key words. galaxies: active - X-rays: galaxies - ISM: clouds

\section{Introduction}

The selection of active galactic nuclei in the hard X-ray band (up to about $10 \mathrm{keV}$ with the XMM-Newton and Chandra satellites), together with their redshift $z$ measurement after optical identification, immediately yields the spectroscopic estimate of the photoelectrically absorbing column density, $N_{\mathrm{H}}$, intrinsic to each of them. A schematic split between objects with $N_{\mathrm{H}}$ either smaller or larger than $10^{22} \mathrm{H} \mathrm{cm}^{-2}$ corresponds fairly well (although not exactly one-to-one) with the optical classification of AGN as either type 1 (broad-line) or type 2 (narrow-line) (see, e.g., Perola et al. 2004). Among the objects with $N_{\mathrm{H}}$ larger than $10^{22} \mathrm{H} \mathrm{cm}^{-2}$, a further subdivision is necessary, those with $N_{\mathrm{H}}$ smaller than $\sigma_{\mathrm{T}}^{-1}=1.5 \times 10^{24} \mathrm{~cm}^{-2}$, called Compton-thin, and those with $N_{\mathrm{H}}>\sigma_{\mathrm{T}}^{-1}$, called Compton-thick. The latter category is hardly represented in samples extracted from images with maximum X-ray energy about $10 \mathrm{keV}$; therefore, the investigations on the fraction of absorbed AGN do in fact apply only to the Comptonthin sources: $f_{\mathrm{abs}}=n\left(10^{22} \leq N_{\mathrm{H}} \leq 10^{24}\right) / n$ (total).

The empirical results for this fraction, reported in the literature, have not yet reached full agreement. To first mention the two papers (Ueda et al. 2003; La Franca et al. 2005) they addressed the issue of $f_{\text {abs }}$ in detail simultaneously with that of the AGN luminosity function in $L_{X}(2-10 \mathrm{keV}), L_{X}$ hereafter, and its cosmological evolution, they agree on the existence of an anticorrelation between $f_{\text {abs }}$ and $L_{X}$, but not on its behaviour with $z$. While its normalization and shape stay constant, according to Ueda et al. (2003), according to La Franca et al. (2005, LF2005), $f_{\text {abs }}\left(L_{\mathrm{X}}\right)$ increases with $z$ and becomes shallower, so that the increase is more marked at high than at low luminosities. Akylas et al. (2006) confirm the existence of the anti-correlation with $L_{\mathrm{X}}$, but argue that the apparent increase in $f_{\text {abs }}$ with $z$ in their data sample might be due to a systematic overestimate of $N_{\mathrm{H}}$ at high redshifts. Evidence of the anti-correlation with $L_{X}$ has also been reported by Hasinger (2004) and by Treister \& Urry (2005; see also Barger et al. 2005). To avoid the uncertainties associated with the estimate of $N_{\mathrm{H}}$, Treister \& Urry (2006) use the optical spectroscopic classification of the counterparts in a combination of seven X-ray-selected samples and claim evidence (after correcting for selection bias) of an increase with $z$ of the fraction of obscured AGN. They do not, however, explicitly address the issue of whether the shape of the anti-correlation might change as well. A correlation between the fraction of broad-line AGN and their luminosity is reported by Simpson (2005), after analysing an optically selected sample $(z<0.3)$ and using the [OIII] narrow emission line to indicate the bolometric luminosity.

Although Dwelly \& Page (2006), after a detailed study of one X-ray deep and narrow beam survey, find no evidence of both the anti-correlation and of a redshift dependence in $f_{\text {abs }}\left(L_{\mathrm{X}}\right)$, we consider it reasonable at present to adopt the view that the existence of the anti-correlation between $f_{\text {abs }}$ and $L_{\mathrm{X}}$ is fairly well-supported. For X-ray-selected samples in particular, we regard the results as more trustworthy that are based on the combination of deep-narrow and shallow-wide surveys (see LF2005 for a discussion on this point). On the other hand, we regard the evidence of an increase in $f_{\mathrm{abs}}\left(L_{\mathrm{X}}\right)$ as still 
Table 1. The parameters used out to $z=0.1$, inferred from $\mathrm{H} 2004$.

\begin{tabular}{cccccccc}
\hline \hline $\log M_{\mathrm{BH}}\left(M_{\odot}\right)$ & $\log L_{\text {bol, },}(\mathrm{erg} / \mathrm{s})$ & $\lambda_{0}$ & $q_{\mathrm{H}}\left(\lambda>\lambda_{0}\right)$ & $\lambda_{\max }$ & $\lambda_{\text {peak }}$ & $w$ & $q_{\mathrm{HI}}\left(\lambda>\lambda_{0}\right)$ \\
\hline 7 & 44.8 & 0.5 & $0.32 \times 10^{-3}$ & 1 & 0.035 & 0.053 & $0.41 \times 10^{-3}$ \\
7.5 & 44.8 & 0.16 & $1.44 \times 10^{-3}$ & 0.8 & 0.006 & 0.02 & $1.53 \times 10^{-3}$ \\
8 & 44.8 & 0.05 & $2.3 \times 10^{-3}$ & 0.4 & 0.005 & 0.012 & $2.3 \times 10^{-3}$ \\
8.5 & 44.8 & 0.016 & $1.26 \times 10^{-3}$ & 0.13 & 0.0005 & 0.004 & $1.26 \times 10^{-3}$ \\
9 & 44.8 & 0.005 & $0.17 \times 10^{-3}$ & 0.04 & 0 & 0.0003 & $0.17 \times 10^{-3}$ \\
\hline
\end{tabular}

controversial with increasing $z$, even more so that of a change in its "slope". In a previous paper (Lamastra et al. 2006a, PapI), we proposed a simple model to explain the anti-correlation, which calls into play interstellar gas for the Compton-thin sources, while the molecular torus, as invoked in the standard "unification" model (Antonucci 1993), remains the best explanation for the Compton-thick sources. Our proposal is no more than an extension of the standard model, whose best empirical support is the finding by Maiolino \& Rieke (1995) of a correlation between absorption and disc inclination in (optically classified as) intermediate type Seyfert galaxies, which is absent in extreme type 2 Seyferts. A finding whose simplest explanation is that the torus and the disc are, in general, not necessarily coplanar. The model assumes that the obscuring matter is distributed in a rotationally supported disk, extending out to a few hundred of parsec, as observed in nearby spiral galaxies mainly in the form of molecular hydrogen. The gravitational pull of the central supermassive black hole (SMBH), combined with that of the bulge (see Ferrarese \& Ford 2005, for a review of the connections between these two galactic components) on the gaseous disc, as a function of the distance $R$ from the centre, reduces the gaseous disc covering factor $C$ as the SMBH mass, $M_{\mathrm{BH}}$, increases. This effects leads to prediction of an anti-correlation between $f_{\text {abs }}$ and $M_{\mathrm{BH}}$, which is likely to be reflected into the one observed between $f_{\text {abs }}$ and $L_{X}$. In PapI it was shown that the latter could be reproduced fairly well in the "local" universe by adopting a "typical" value of 0.1 , irrespective of the mass, of the ratio between the bolometric and the Eddington luminosity, $\lambda=L_{\mathrm{b}} / L_{\mathrm{E}}$. This value of $\lambda$ was consistent with the estimates available then (see references in PapI).

Recently, by means of a wealth of spectroscopic data from the Sloan Digital Sky Survey (SDSS), the empirical knowledge of the behaviour of $\lambda$ as a function of $M_{\mathrm{BH}}$ has had a substantial turn, thanks in particular to the work by Netzer \& Trakhtenbrot (2007, NT2007) on broad-line AGN. They explored it for masses from $10^{7}$ to $10^{9} M_{\odot}$ in the $z$ interval $0.3-0.7$, and found a strong dependence on both $M_{\mathrm{BH}}$ and $z$. It should be noted that several earlier papers seemed to agree on a practically constant typical value of $\lambda$ with increasing $z$, irrespective of luminosity and mass, after selection effects had been taken into account (a good example, over the range $z=0.3-4$ in Kollmeier et al. 2006; see also Lamastra et al. 2006b). In the framework of a model where, by definition, the intrinsic properties of the central engine are the same in broad and narrow-line objects, it is natural to assume that the behaviour found by NT2007 holds for the narrow-line AGN as well. As a matter of fact, in the "local" (around $z=0.1$ ) universe, a qualitatively similar result for $\lambda\left(M_{\mathrm{BH}}\right)$ was found earlier on by Heckman et al. (2004, H2004) in a large sample of SDSS selected narrow-line AGN. Despite some criticism of the reliability of the methods used by $\mathrm{H} 2004$ expressed by Netzer et al. (2006), we use their findings as well.

The new light on the $M_{\mathrm{BH}}-\lambda-z$ relationships in the range of $z$ up to 0.7 prompted us to revisit the model of PapI, with the further aim of verifying whether the $z$-dependence of $f_{\mathrm{abs}}$ claimed by LF2005 might be reproduced or otherwise.

This paper is organised as follows. Section 2 is devoted to the new results mentioned above and to their quantitative representation as is used later, Sect. 3 to the mass function of the $\mathrm{SMBH}$, Sect. 4 to the molecular gas surface density in the inner region of galaxies, both late and early-types. Sections 5 and 6 present the model "predictions" of $f_{\mathrm{abs}}$ as a function of $L_{\mathrm{bol}}$ and of $L_{\mathrm{X}}$, and their comparison with the LF2005 results. A discussion and a conclusion follow in Sects. 7 and 8.

\section{New light on the $M_{\mathrm{BH}}-\lambda-z$ relationships}

NT2007 uses a sample of almost ten thousand broad-line AGN, with $m_{i} \leq 19.1$, from the SDSS to investigate the distribution of $\lambda$ in different range values of $M_{\mathrm{BH}}$, from $10^{7}$ to $10^{9} M_{\odot}$, as a function of the redshift $z$. They estimate $M_{\mathrm{BH}}$ using the $L_{5100}-R_{\mathrm{BLR}}$ (the radius of the broad-line region) relationship (as given by Kaspi et al. 2005) in combination with the $\mathrm{FWHM}(\mathrm{H} \beta)$. The value of $\lambda$ is obtained after applying a (constant) bolometric correction to $L_{5100}$. The results, as summarised in their Table 1 , apply to intervals of $z$ up to $z_{\max }=0.75$, except for the mass range around $10^{7} M_{\odot}$ for which $z_{\max }=0.3$, and down to $z_{\min }=0.05$ for the mass ranges around $10^{7}$ and $10^{7.5} M_{\odot}$, with $z_{\min }$ increasing up to 0.25 for the mass range around $10^{9} \mathrm{M}_{\odot}$. Thus, in the $z$ interval $0.25-0.75$, the results apply to all masses from $10^{7.5}$ to $10^{9} M_{\odot}$, while the overlap is confined to the narrow $z$ interval $0.25-0.3$ for the mass range around $10^{7} M_{\odot}$. These results concern a feature, $\lambda_{\text {peak }}$, defined as the maximum in the observed $\lambda$-distributions, and its dependence on $M_{\mathrm{BH}}$ and on $z$. NT2007 apply reliable statistical tests to demonstrate that the dependence found is most likely real, rather than a consequence of the flux limit of the sample.

To avoid misunderstandings on the extent to which we rely on NT2007 in the following, it is appropriate to comment on the "incompleteness" of their sample. As extensively discussed in particular by Richards et al. (2006), whose aim was to estimate the luminosity function of QSO as a function of the redshift, samples of such objects from the SDSS are affected by incompleteness due to the target selection algorithm, based on the observed photometric colors, and to the limiting magnitude. From the statements in NT2007, we infer that the value of $\lambda$ (see the marks in their Fig. 3, call it $\lambda_{\text {mark }}$ ), below which they regard their sample incomplete, relates to the limiting magnitude $m_{i} \leq 19.1$. Figure 6 in Richards et al. (2006) shows clearly that the incompleteness degree increases very steeply for magnitudes above this limit. In the same figure it is apparent that the incompleteness due to the color selection is of the order of $20 \%$ (averaged over $15<m_{i}<19.1$ ) and stays constant up to $z$ about 2 . Going through the way Richards et al. (2006) then used these results to obtain the proper normalization of the luminosity function, we are led to conclude that the color-induced selection effects do impact on the "absolute" values, not on the "relative" ones. In other words, the "shape" of the distributions of $\lambda$ above $\lambda_{\text {mark }}$ 
should not be affected by these effects. Since for our purpose, as will be apparent later, it is the "shape" of these distributions that is essential, we now describe the way we have adopted to quantify this shape.

We introduce the distribution function $\Phi\left(M_{\mathrm{BH}}, \lambda, z\right)$. Figure 3 in NT2007 displays the fraction of AGN as a function of $\lambda$ for two ranges of black hole mass $\left(10^{7.5}-10^{7.8} ; 10^{8.5}-10^{8.8} M_{\odot}\right)$, each one for three values of $z$. Except for the range $M_{\mathrm{BH}}=$ $10^{7.5}-10^{7.8} M_{\odot}$ at $z=0.5, \lambda_{\text {mark }}$ is placed somewhat to the left of the peak in the "observed" distribution in the other five cases. We thence looked for an analytical function that could represent the five "observed" distributions above $\lambda_{\text {mark }}$ reasonably well. We eventually adopted a Lorentzian profile:

$\Phi\left(M_{\mathrm{BH}}, \lambda, z\right)=\frac{A\left(M_{\mathrm{BH}}, z\right)}{2 \pi} \frac{w}{\left(\lambda-\lambda_{\text {peak }}\right)^{2}+w^{2}} \quad \lambda>\lambda_{\text {mark }}$,

with a "natural" cut-off at $\lambda=1$.

After assigning statistical error bars corresponding to a total number of 150 objects (a relatively arbitrary choice between the total numbers from 50 to 230 declared in NT2007 without further specifications) to each one of the five histograms in NT2007, Fig. 3, we operated a $\chi^{2}$ fit to obtain the five values of $\lambda_{\text {peak }}$ and of $w$. After noting that all five values of $w$ turned out to be equal to $\lambda_{\text {peak }}$ multiplied by approximately the same constant, we proceeded with a simultaneous fit after requiring that this constant should be exactly the same. The result obtained is $w=1.5 \lambda_{\text {peak }}$, with a reduced $\chi^{2}=0.68$. We then adopted this result to $w$ to hold for any mass value from $10^{7}$ to $10^{9} M_{\odot}$, independent of $z$. It is to be noted, at last, that our values of $\lambda_{\text {peak }}$ do not differ significantly from those, apparently obtained by NT2007 directly from the position of the peak in their fractional distributions, displayed in their Fig. 4. (We later return on the normalization $A\left(M_{\mathrm{BH}}, z\right)$.)

For the $z$-dependence of $\lambda_{\text {peak }}\left(M_{\mathrm{BH}}\right)$, NT2007 find (see also their Fig. 4) that it can be represented (of course only in the intervals of $z$ effectively covered) with a power law, namely:

$\lambda_{\text {peak }} \propto z^{\gamma\left(M_{\mathrm{BH}}\right)}$

where the exponent $\gamma$ increases with $M_{\mathrm{BH}}$. We therefore adopt the following $z$-dependence:

$\log \lambda_{\text {peak }}\left(M_{\mathrm{BH}}, z\right)=\log a\left(M_{\mathrm{BH}}\right)+\gamma\left(M_{\mathrm{BH}}\right) \log z$.

For our application, it is convenient to assign an analytical form to the function $\gamma\left(M_{\mathrm{BH}}\right)$ and $a\left(M_{\mathrm{BH}}\right)$. For the former, it turns out that the five values of $\gamma$ in NT2007, Table 1, are well-fitted with

$\gamma\left(M_{\mathrm{BH}}\right)=-3.01+0.58 \log \left(M_{\mathrm{BH}}\right)$.

For $a\left(M_{\mathrm{BH}}\right)$, which is not given in NT2007, we have to rely upon the only two values obtained with the above-mentioned fits. We choose to connect them with a straight line in the log-log plot of $a$ and $M_{\mathrm{BH}}$, and obtained

$a\left(M_{\mathrm{BH}}\right)=10^{5.5}\left(M_{\mathrm{BH}} / M_{\odot}\right)^{0.74}$.

The power-law relationship (3) is illustrated in Fig. 1 for five representative mass values, from $10^{7}$ to $10^{9} M_{\odot}$. Except for $M_{\mathrm{BH}}=10^{7} M_{\odot}$, the normalization comes from (5). The extrapolation of Eq. (5) to this mass places the power law well above the three $\lambda_{\text {peak }}(z)$ points in NT2007, Fig. 4, which are reported in our Fig. 1. We therefore decided to adopt a power law with a slope as given by Eq. (4) and a normalization in agreement with these points. The figure illustrates very well that the power law (3) pertaining to a given mass always stays above the one pertaining to the next higher mass.

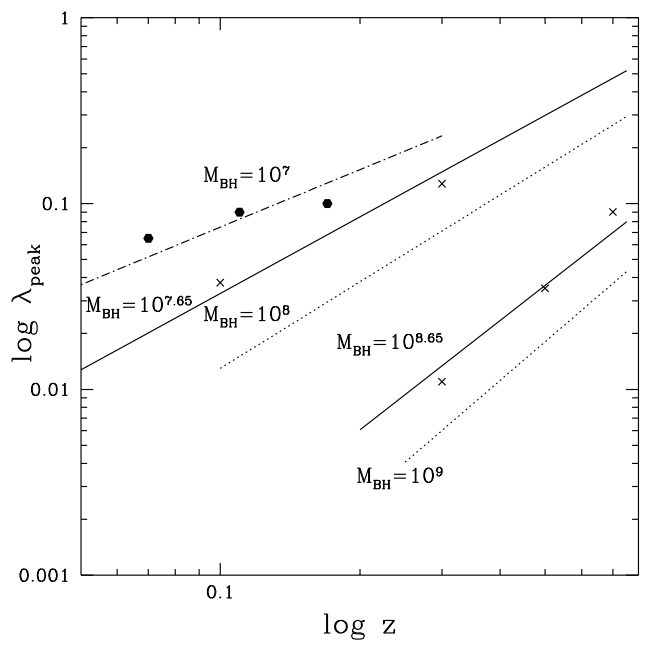

Fig. 1. The redshift dependence of $\lambda_{\text {peak }}$ for five values of $M_{\mathrm{BH}}$ in $M_{\odot}$, derived from NT2007 as described in the text. The crosses are the values obtained by fitting the Lorentzian profile (1), the hexagons are from Fig. 4 in NT2007.

To use the function $\Phi$, there are three other elements to be taken into consideration. The first would be the extrapolation of $\Phi$ below $\lambda_{\text {peak }}\left(M_{\mathrm{BH}}, z\right)$. This is extremely uncertain, so we avoided even trying. We exploit the fortunate circumstance that the information available on the shape of $\Phi$ above $\lambda_{\text {peak }}$ will allow us (see Sects. 5 and 6) to predict the quantity $f_{\text {abs }}$ for $L_{\mathrm{bol}} \geq 10^{45} \mathrm{erg} \mathrm{s}^{-1}$ (corresponding to $L_{\mathrm{x}} \simeq 10^{43.5} \mathrm{erg} \mathrm{s}^{-1}$, Marconi et al. 2004), namely the range of $L_{\mathrm{x}}$ where the increase in $f_{\text {abs }}$ with $z$, is most pronounced according to LF2005. Incidentally, this is also the luminosity interval where the SDSS quasar luminosity function was determined by Richards et al. (2006); see also Hopkins et al. (2007).

The second is the normalization $A\left(M_{\mathrm{BH}}, z\right)$. Since we use $\Phi$ only for $\lambda \geq \lambda_{\text {peak }}$, it is convenient to introduce the integral quantity

$\int_{\lambda_{\text {peak }}}^{1} \Phi\left(M_{\mathrm{BH}}, \lambda, z\right) \mathrm{d} \lambda=q\left(M_{\mathrm{BH}}, z\right)$.

This quantity, which depends on $A\left(M_{\mathrm{BH}}, z\right)$, represents the fraction of $\mathrm{SMBH}$, as a function of their mass and cosmological epoch, which are active above $\lambda_{\text {peak }}$.

There are no elements in NT2007 to infer the value of $q$. For our purpose, however, what matters is whether this fraction is either a constant or a function of $M_{\mathrm{BH}}$; in other words, whether it preserves the same shape as the active $\mathrm{SMBH}$ mass function ( $q=$ const.) or not ( $q$ depends on $\left.M_{\mathrm{BH}}\right)$. In Sect. 5 we illustrate the consequences of both types of assumption.

The third is the mass function (MF) of the SMBH in galaxies, $F\left(M_{\mathrm{BH}}\right)$, in particular that of the active ones. The MF is needed to weight the fractional function $\Phi$ when the contributions to $f_{\mathrm{abs}}$ by different masses are combined within the same bin of luminosity. The MF will be dealt with in Sect. 3 .

We lack a similar analysis for $\lambda\left(M_{\mathrm{BH}}, z\right)$ carried out explicitly on broad-line AGN in the low-redshift universe. Thence we resorted to the work by $\mathrm{H} 2004$ on a large (23000) sample of narrow-line AGN drawn from the SDSS. To the extent that this other type of AGN can be assumed to share the same intrinsic properties of the broad-line type, we can confidently use their results to include the local (out to $z=0.1$ ) universe in our work.

Unlike NT2007, H2004 estimate the mass using the $M_{\mathrm{BH}}-$ $\sigma^{*}$ (stellar velocity dispersion in the galaxy bulge) relationship 


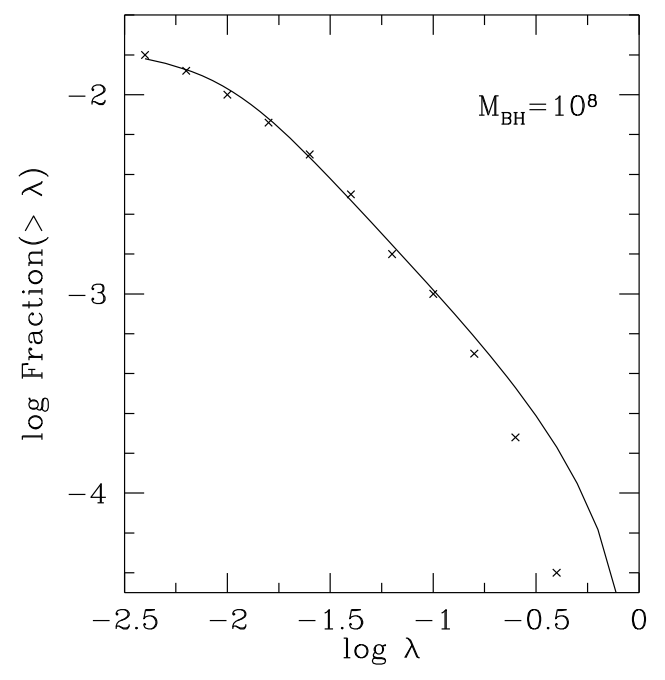

Fig. 2. Integral distribution in $\lambda$ of the active fraction of SMBH, $M_{\mathrm{BH}}=$ $10^{8} M_{\odot}$, out to $z=0.1$. The crosses are derived from H2004, Fig. 3, the line is our Lorentzian representation.

(as given by Tremaine et al. 2002). The need to measure $\sigma^{*}$ practically excluded from the sample the brightest Seyfert galaxies and the QSO, a cautionary remark emphasised by H2004. They derive $L_{\text {bol }}$ from $L_{\text {OIII }}$ multiplied by a constant factor $(3500$, estimated variance of $0.38 \mathrm{dex}$ ). This procedure is not completely reliable, because there seems to be a correlation between the correction factor and the luminosity (Netzer et al. 2006), but we regard it as acceptable in the first approximation for our purpose.

The quantitative results that we use are those summarised in H2004, Fig. 3 (left panel), where the integral distribution in $\lambda$ of the fraction of $\mathrm{SMBH}$, which are active, is given for six values of $M_{\mathrm{BH}}$, from $3 \times 10^{6}$ to $10^{9} M_{\odot}$. It can be immediately seen that, at least qualitatively, the trend of $\lambda$ with $M_{\mathrm{BH}}$ is the same as found by NT2007. This form of presentation, namely the fraction of active objects with respect to the "local" $F\left(M_{\mathrm{BH}}\right)$, is particularly convenient because it can be directly used, as we do in Sect. 6, without resorting to assumptions on the quantity $q$, Eq. (6). It is also interesting to check whether these distributions can be represented by the function (1). Since the curves in H2004, Fig. 3, are devoid of error bars, we could only adjust, as best as we could, the integral of this function to them. The outcomes are in general quite satisfactory, and one example is shown in Fig. 2 (where the curve from H2004 is represented by a selection of points). Typically the high $\lambda$ tails stay somewhat above the $\mathrm{H} 2004$ curves. One might require that the excess in the Lorentzian tail does to some extent take care of the lack of the brightest objects in the original sample, but we need not insist on this issue, because the difference is of little consequence for our final results.

The parameters extracted from $\mathrm{H} 2004$ that we use in Sect. 6 are summarised in Table 1 . The $L_{\mathrm{bol}, 0}$ in the second column (with the corresponding $\lambda_{0}$ in the third) represents a completeness limit valid for all masses between $10^{7}$ and $10^{9} M_{\odot}$. The fourth and fifth columns contain, repectively, $\lambda_{\max }$, the maximum value that can be read in $\mathrm{H} 2004$, Fig. 3, and $q_{\mathrm{H}}$, the fraction of active SMBH above $\lambda_{0}$ from the same figure. From the sixth column onward the quantities given were obtained using the Lorentzian profile, as explained above. The quantity $\lambda_{\text {peak }}$ is very low and badly determined, but the quantity that really matters is $w$; the fraction $q_{\mathrm{Hl}}$ in the last column differs only marginally from $q_{\mathrm{H}}$.

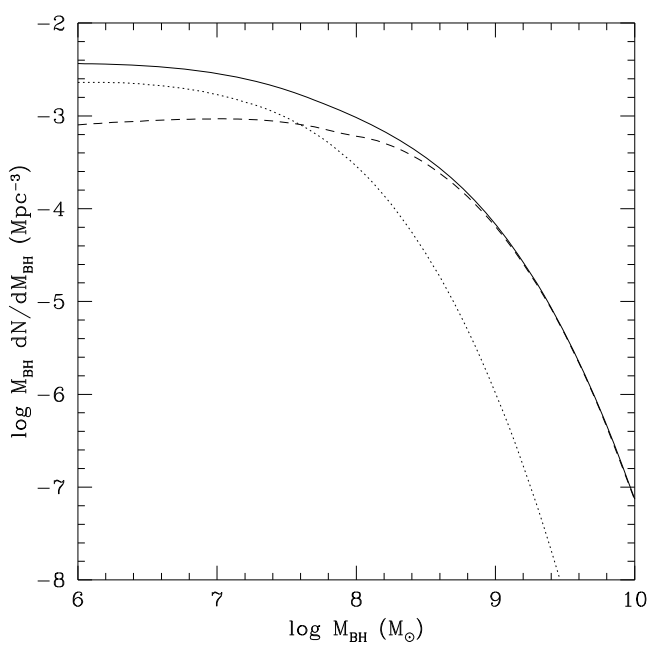

Fig. 3. The local SMBH mass function from Marconi et al. (2004). Dotted line: late type galaxies; broken line: early type galaxies.

It should be noted that the fraction of active SMBH in $\mathrm{H} 2004$ is given with respect to the local $F\left(M_{\mathrm{BH}}\right)$, such as the one given by Marconi et al. (2004) and described in the next section.

\section{The mass function of SMBH}

The number density of SMBH as a function of mass, $F\left(M_{\mathrm{BH}}\right)$, has been estimated in the local universe by several authors (see Marconi et al. 2004; Shankar et al. 2004, and references therein). The one adopted here is from Marconi et al. (2004) and is shown in Fig. 3, where the MF which includes all morphological types (their Fig. 2b) is given along with the MF in late, $F_{\mathrm{L}}$, and early, $F_{\mathrm{E}}$, type galaxies separately (Marconi, priv. comm.). This $F_{\mathrm{L}}$ is preferred to the one estimated by Shankar et al. (2004) for the attention paid to the change in the bulge to the total luminosity ratio along the Hubble sequence of late type galaxies. The reason we keep the late separate from the early type galaxies will be explained in Sect. 7.

For our purpose, what does matter is the shape of the MF. Insofar as the growth of the SMBH is a consequence of accretion and is therefore accompanied by the AGN type activity, it is most likely that the shape of the MF for the "active" SMBH changes with redshift and is different from that of the whole population as observed in the local universe. In principle, the redshift dependence of the "active" SMBH mass function, $F^{*}\left(M_{\mathrm{BH}}, z\right)$, could be inferred from the AGN luminosity function as a function of $z$, provided that a simple relationship holds between $M_{\mathrm{BH}}$ and the luminosity (for instance, $\lambda=$ const.). In practice, as discussed in Marconi et al. (2004) and as has become particularly evident after NT2007, the conversion is neither straightforward nor univocal. Hence $F^{*}\left(M_{\mathrm{BH}}\right)$ can only be reliably estimated through a "direct" evaluation of $M_{\mathrm{BH}}$ in a properly selected sample of AGN, with special care devoted to correcting for incompleteness selection effects. On the basis of SDSS samples, H2004 $(z \leq 0.1)$ for narrow emission line AGN and Greene \& Ho (2007, $z \leq 0.3$, with masses derived as in NT2007) for broad emission line AGN, both find that in the local universe $F^{*}\left(M_{\mathrm{BH}}\right)$ is steeper, above $10^{7} M_{\odot}$, than $F\left(M_{\mathrm{BH}}\right)$ : a difference attributed to the so-called downsizing

In Sects. 5 and 6 we, in a first instance, adopt the "shape" of the local $F\left(M_{\mathrm{BH}}\right)$; however, the impact on the results of other options for the downsizing is also discussed. 


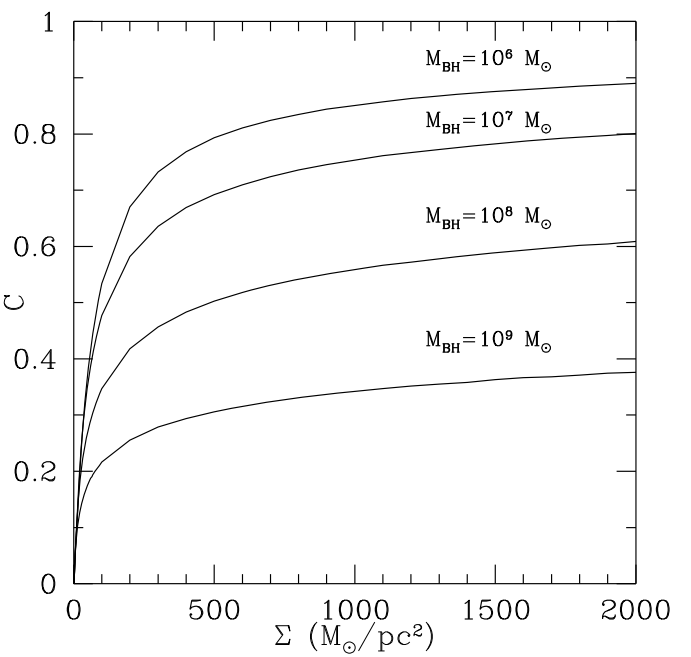

Fig. 4. The covering factor $C$ as a function of $\Sigma$, according to the model, for different values of the SMBH mass. From top to bottom: $10^{6}, 10^{7}$, $10^{8}$, and $10^{9} M_{\odot}$.

\section{The molecular gas surface density $\Sigma$}

The basic feature of the model in PapI is the gravitational force exerted by the $\mathrm{BH}$ (and by the associated stellar bulge) on a rotationally supported disc made of molecular clouds. This force shapes the gas distribution profile, as a function of the radial distance $R$ from the $\mathrm{BH}$, in such a way as to determine an anticorrelation between $M_{\mathrm{BH}}$ and the "covering factor" determined by the disc. Quantitatively this factor was calculated in PapI assuming that the distance out to which the disc extends is at least equal to $2 R_{\text {infl }}\left(R_{\text {infl }}\right.$ is the distance from the $\mathrm{BH}$ at which the molecular gas distribution profile has a point of inflection, see Eq. (9) in PapI), that is, about $25 \mathrm{pc}$ when $M_{\mathrm{BH}}=10^{6}$ and extending to about 450 pc when $M_{\mathrm{BH}}=10^{9} M_{\odot}$.

Figure 4 shows this "covering factor", $C\left(\Sigma, M_{\mathrm{BH}}\right)$, as a function of the surface density $\Sigma$, from 0 to $2000 M_{\odot} \mathrm{pc}^{-2}$, for four values of $M_{\mathrm{BH}}$, from $10^{6}$ to $10^{9} M_{\odot}$. After a steep growth, the curves enter a regime where the increase is very slow. The rather sharp change in slope takes place around a $\Sigma$ value from about 200 to about $400 M_{\odot} \mathrm{pc}^{-2}$ when moving down from $M_{\mathrm{BH}}=10^{9}$ to $M_{\mathrm{BH}}=10^{6} M_{\odot}$. Thus, in predicting $f_{\mathrm{abs}}$, attention must be paid to the distribution of $\Sigma$ adopted, particularly on the fraction of objects below those values.

In PapI (to which we refer the reader for more details), we used the only results obtained with sufficient angular resolution in a molecular line. These are represented by a sample of 44 nearby and bright spiral galaxies mapped at the $3 \mathrm{~mm} \mathrm{CO} J=$ 1-0 line, the BIMA SONG survey (Helfer et al. 2003). At their average distance of $12 \mathrm{Mpc}$, the angular resolution of 6 arcsec corresponds to a radial distance from the centre of about $210 \mathrm{pc}$.

In Fig. 5 the central face-on $\Sigma$ of these objects is plotted as a function of the mass of the SMBH (estimated as explained in the figure caption). For the majority of these galaxies $M_{\mathrm{BH}}$ is comprised between $10^{6.5}$ and $10^{8.5} M_{\odot}$. In practice this is the range of masses for which the information described in Sect. 2 will be used to predict $f_{\text {abs }}$ in Sects. 5 and 6. Given the small number of objects in this sample, we use the observed distribution of $\Sigma$ in the 44 objects to evaluate the uncertainty on $f_{\text {abs }}$.

In PapI we stressed that the effect under study does not require any type of correlation between the central activity and the presence of the absorbing gas, the latter being "in place" as a property of the galactic interstellar medium. This statement

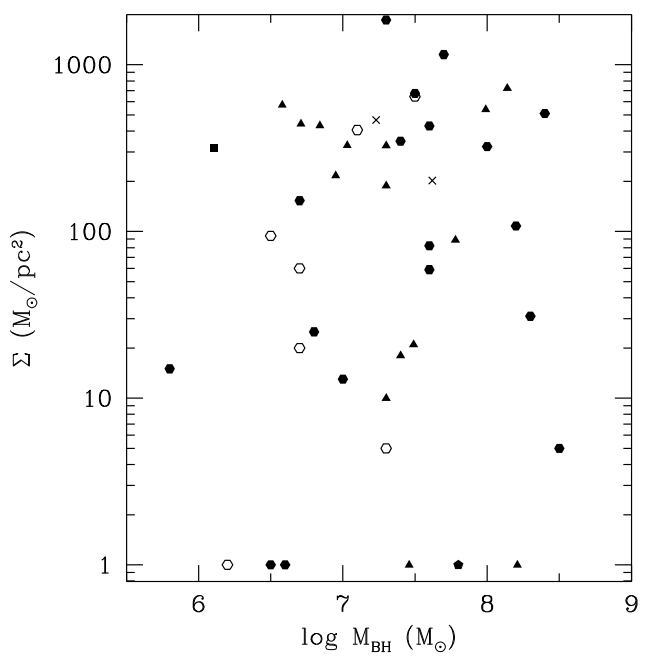

Fig. 5. The molecular gas central, face-on surface density $\Sigma$ of the spiral galaxies in the BIMA SONG survey, versus the mass estimate of the SMBH hosted by these galaxies. Hexagons: $M_{\mathrm{BH}}$ from the $M_{\mathrm{BH}}-L_{\text {bulge }}$ relationship; open: from Dong \& De Robertis (2006); filled: our work. Triangles: from the $M_{\mathrm{BH}}-\sigma^{*}$ relationship, Batcheldor et al. (2005), Merloni et al. (2003), Woo \& Urry (2002), Pellegrini (2005). Crosses and pentagon: from maser and from stellar kinematics, Ho (2002). Square: from reverberation mapping, Kaspi et al. (2000).

can be regarded as basically correct for the late type galaxies (not only in the local universe, as shown by the survey just mentioned, but presumably also at cosmological distances), and now we need to consider to what extent it might be applied to the early type galaxies.

Elliptical (E) and lenticular (S0), unlike the spiral (S) galaxies, are rare in the field and frequent (the bright E's in particular) in galaxy groups and clusters. Therefore, useful interferometric observations are only available for a dozen of them, optically luminous or very luminous (thus hosting SMBH well above $10^{7} M_{\odot}$ ), out to a maximum distance of about $85 \mathrm{Mpc}$ and with a strong CO line signal. It is convenient to concentrate first on these results, and we limit ourselves to those obtained by Young $(2002,2005)$ with the BIMA interferometer on seven "true" E (that is galaxies with an $r^{1 / 4}$ photometric profile). Molecular gas is found distributed in rotating, mostly symmetric discs, with radii ranging from 1 to $6 \mathrm{kpc}$. For each of them we report the peak surface brightness, translated into a surface density of $\mathrm{H}_{2}$ gas, along with the galaxy surface area corresponding to the beam. UGC 1503 (field): $60 M_{\odot} \mathrm{pc}^{-2}$ $\left(2.4 \times 2.1 \mathrm{kpc}^{2}\right)$; NGC 807 (field): $40 M_{\odot} \mathrm{pc}^{-2}\left(2.4 \times 2.0 \mathrm{kpc}^{2}\right)$; NGC 3656 (merger remnant): $720 M_{\odot} \mathrm{pc}^{-2}\left(1.7 \times 1.3 \mathrm{kpc}^{2}\right)$; NGC 4476 (Virgo cluster): $112 M_{\odot} \mathrm{pc}^{-2}\left(0.73 \times 0.49 \mathrm{kpc}^{2}\right)$; NGC 5666 (field): $115 M_{\odot} \mathrm{pc}^{-2}\left(1.7 \times 1.3 \mathrm{kpc}^{2}\right)$ (from Young 2002). NGC 83 (group): $135 M_{\odot} \mathrm{pc}^{-2}\left(2.7 \times 2.5 \mathrm{kpc}^{2}\right)$; NGC 2320 (A569): $353 M_{\odot} \mathrm{pc}^{-2}\left(2.4 \times 2.2 \mathrm{kpc}^{2}\right)$ (from Young 2005). On account of the values of the area/beam, it is evident that these selected cases correspond to the values of $\Sigma$, which imply a substantial covering factor $C$ and which occur in a large fraction of the $\mathrm{S}$ galaxies in Fig. 5. But how frequent the occurrence is of such a situation in E and S0 galaxies? The answer to this question does not appear to be well settled yet. Furthermore, most data are from single-dish observations, which can be properly converted only into a total $\mathrm{H}_{2}$ mass.

On this issue it seems to us appropriate to first quote the compilation by Bettoni et al. (2003) of a wealth of data obtained prior to the year 2003. Most useful for our purpose are this mean 
sample values, subdivided per morphological class according to the mean numerical Hubble stage index $t$ as tabulated in the Third Reference Catalogue of Bright Galaxies (RC3 de Vaucouleurs et al. 1991), of the quantity $M\left(\mathrm{H}_{2}\right) / D_{25}^{2}$, where $D_{25}$ is the linear size corresponding to the $25 \mathrm{mag} / \operatorname{arcsec}^{2}$ isophote. After assembling their classes into three groups, $\mathrm{G} 1$ ( $t$ from -5 to $-3, \mathrm{E}), \mathrm{G} 2$ ( $t$ from -2 to $0, \mathrm{~S} 0$ ), and $\mathrm{G} 3$ ( $t$ from 1 to $6, \mathrm{Sa}$ to Sc), from Table 5 in Bettoni et al. (2003), we obtain the following proportions for the quantity $M\left(\mathrm{H}_{2}\right) / D_{25}^{2}, \mathrm{G} 1: \mathrm{G} 2: \mathrm{G} 3=$ $0.06: 0.29: 1$. Thus, with the cautionary remark that the size of the $\mathrm{H}_{2}$ gas structure in the three groups may not scale similarly with $D_{25}$, the immediate conclusion one can draw is that in the ellipticals the surface density of molecular gas is, on average, more than one order of magnitude less than in spirals, while it is a factor of about three less in the lenticulars. This conclusion we believe is stronger than the one that can be drawn from the detection rate, which is very sensitive to the sample selection, as discussed in Combes et al. (2007); see also Welch \& Sage (2003), Sage et al. (2007) and references in these papers. An additional important result emerging from these papers is that, among the early type galaxies, the ratio $M\left(\mathrm{H}_{2}\right) / L$ decreases with increasing luminosity $L$, despite the increase in depth of the potential well. Taken together with the results quoted from Bettoni et al. (2003), this finding leads us to conclude that the more luminous (and therefore hosting the more massive SMBH) an early type galaxy, the less the chance of finding "in place" $\mathrm{H}_{2}$ surface densities similar to the ones reported above from Young (2002, 2005).

For high $z$ objects, we would like to mention the most obvious argument used to account for the $M_{\mathrm{BH}}-\sigma^{*}$ and $M_{\mathrm{BH}}-L$ observational relationships (see Ferrarese \& Ford 2005, for a review), where $\sigma^{*}$ and $L$ are the stellar velocity dispersion and the luminosity, respectively, of galactic "bulges" or "spheroids". The argument goes that the formation of the spheroids is closely connected with the growth of the SMBH sitting at their centers. If the two phenomena had progressed hand-in-hand from the earliest to the present times, it would be legitimate to postulate that a phase of intense activity (AGN phase) should in general be accompanied by star formation on the spatial scale of the bulge. The latter requires the accumulation on this scale of gas (whatever its origin) in a dense, most likely molecular state, wherein the star formation takes place. From an observational point of view the validity of such a postulate rests on results obtained with studies of vast samples of galaxies, based on SDSS spectrography and photometry. Here we refer in particular to Kauffmann et al. (2003a), Kauffmann et al. (2003b), H2004. Quoting from the review by Heckman \& Kauffmann (2006): "Strong emission-line AGN inhabit those unusual galaxies that are both relatively massive and dense, yet have a significant young stellar population". The denomination "unusual" emphasises the contrast with the usual make-up of galaxies with similar mass and structure, which, within the limits in sharpness of the observational distinctions employed, contain only a population of old stars.

A cautious theoretical elaboration on these results must inevitably contemplate the possibility that the SMBH activity is a consequence of the transformation into stars of the dense gas and the dynamical implications of supernovae explosions in particular. In this instance, it could well be that, during most of the active phase of the $\mathrm{SMBH}$, the dense gas is no longer present in sufficient quantity on the spatial scale required by our model. Furthermore, it is doubtful that the structure of the molecular gas were dominated, in such a phase, even if present, by a rotationally supported configuration out to several $100 \mathrm{pc}$. Only direct observations in molecular lines, with the adequate angular resolution, will answer these questions.

To reach simple and sharp conclusions, we therefore consider two instances. In Sects. 5 and 6, in order to emphasise only the consequences of what was described in Sect. 2, we shall posit that, for all AGN no matter the morphological type of the host galaxies, the model applies equally well and a single representative distribution of $\Sigma$ will be used. In Sect. 7, we posit that the above applies only to the late type galaxies, while in the active early type galaxies the "covering factor" is basically unknown. The latter instance requires that $F_{\mathrm{L}}\left(M_{\mathrm{BH}}\right)$ be kept separate from $F_{\mathrm{E}}\left(M_{\mathrm{BH}}\right)$.

\section{Predicting $f_{\text {abs }}$ as a function of luminosity for $M_{\mathrm{BH}}>10^{7} M_{\odot}$, at $z=0.35$}

The fraction of absorbed AGN, $f_{\text {abs }}$, in a given luminosity bin $\left(L_{\mathrm{b} 1}<L_{\mathrm{b}}<L_{\mathrm{b} 2}\right)$ can be computed using the equation

$$
\begin{aligned}
f_{\mathrm{abs}}= & \left(\sum_{j} \sum_{i} C\left(\Sigma_{j}, M_{\mathrm{BH}, i}\right) f\left(\Sigma_{j}\right) \Delta \Sigma_{j} F\left(M_{\mathrm{BH}, i}\right)\right. \\
& \left.\times \Delta M_{\mathrm{BH}, i} \int_{\lambda_{1}}^{\lambda_{2}} \Phi\left(M_{\mathrm{BH}, i}, \lambda, z\right) \mathrm{d} \lambda\right) \\
& \times \frac{1}{\sum_{i} F\left(M_{\mathrm{BH}, i}\right) \Delta M_{\mathrm{BH}, i} \int_{\lambda_{1}}^{\lambda_{2}} \Phi\left(M_{\mathrm{BH}, i}, \lambda, z\right) \mathrm{d} \lambda}
\end{aligned}
$$

where $\lambda_{1}=\frac{L_{\mathrm{b} 1}}{L_{\mathrm{Edd}}\left(M_{\mathrm{BH}, i}\right)}\left(\lambda_{1} \geq \lambda_{\text {peak }}\right)$ and $\lambda_{2}=\frac{L_{\mathrm{b} 2}}{L_{\mathrm{Edd}}\left(M_{\mathrm{BH}, i}\right)}\left(\lambda_{2} \leq 1\right)$, $f\left(\Sigma_{j}\right)$ is the fractional distribution in the quantity $\Sigma$, introduced to take properly into account the strong dependence of $C(\Sigma)$ on low values of the argument. In our application we used mass bins 0.3 dex wide, and the results are presented in bins of $L_{\mathrm{b}}$ that are 0.5 dex wide. A normalization of the function $\Phi$, as explained in Sect. 2, will depend on the assumption we make on the quantity $q\left(M_{\mathrm{BH}}\right)$ given by Eq. (6).

To predict $f_{\mathrm{abs}}\left(L_{\mathrm{b}}\right)$ with Eq. (7), we only consider the active SMBH with $\lambda>\lambda_{\text {peak }}\left(M_{\mathrm{BH}}\right)$. When the relationshps (3) and (5) are combined, it turns out that, at one particular value of the redshift, $z=0.35, \lambda_{\text {peak }}\left(M_{\mathrm{BH}}\right) \propto M_{\mathrm{BH}}^{-1}$ very closely. Therefore, all masses in the range $10^{7.5}-10^{9} M_{\odot}$, to which at $z=0.35$ both (3) and (5) apply, contribute above the same value of $L_{b}$, equal to $10^{45} \mathrm{erg} \mathrm{s}^{-1}$. In other words, the active $\mathrm{BH}$ in this range of mass with $\lambda<\lambda_{\text {peak }}$ contributes only below $10^{45} \mathrm{erg} \mathrm{s}^{-1}$. Notably, this is the $L_{\text {edd }}$ of $M_{\mathrm{BH}}=10^{7} M_{\odot}$. Thus, to obtain a "complete" estimate of $f_{\mathrm{abs}}$ in the interval of $\log L_{\mathrm{b}}=45-45.5$, only the contribution of the masses in the range $10^{7}-10^{7.5} M_{\odot}$ needs to be added, which we did by resorting to a small extrapolation of the curve labelled $10^{7} M_{\odot}$ in Fig. 1 beyond its formal limit at $z=0.3$. This convenient situation will be exploited to make some clear point, and to predict $f_{\text {abs }}$ at a given $z$ to be used in Sect. 6 for comparison with the predictions at higher and lower values of the redshift.

As anticipated in Sects. 3 and 4, we first adopt the "shape" of the local $F\left(M_{\mathrm{BH}}\right)$, Fig. 3, as representative of the ensamble of active SMBH in both late and early type galaxies, and assume that the "model" applies equally well to the two types of hosts with the same distribution of $\Sigma$. Concerning the quantity $q$ (Eq. (6)), we start with the assumption that it is equal to a constant, namely independent of $M_{\mathrm{BH}}$. It is perhaps worth emphasising that the value of this constant is irrelevant for obtaining the fraction $f_{\mathrm{abs}}$, while its precise knowledge would be necessary if we wanted to predict the number, for instance per unit volume, of the two 


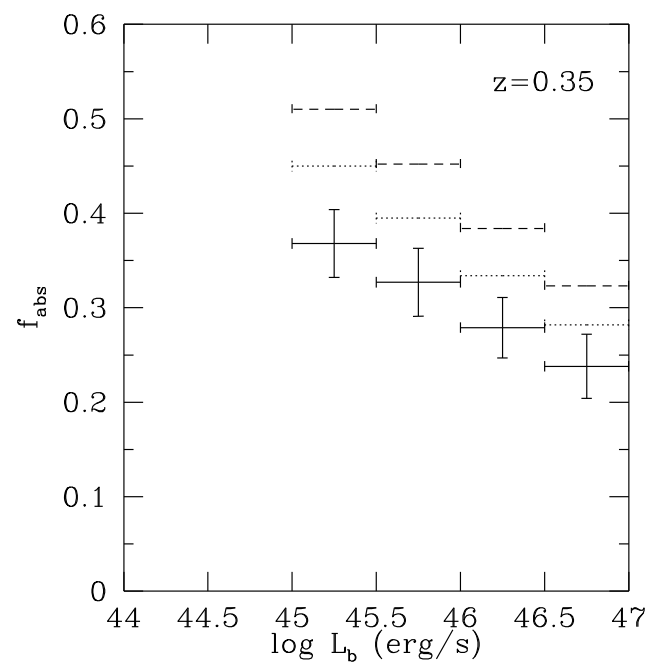

Fig. 6. The predicted $f_{\text {abs }}$ at $z=0.35$, obtained using the local SMBH MF, $q=$ const., with three different choices for $\Sigma$. Full line histogram: the observed distribution of $\Sigma$ described in the text, the bars represent the systematic uncertainty due to the limited number of objects in this distribution. Dotted and broken line histograms: $\Sigma=150$ and $270 M_{\odot} \mathrm{pc}^{-2}$ respectively.

types of AGN. The result is given as a histogram in Fig. 6. The first aspect deserving a comment is the sensitivity of the result to the choice concerning $\Sigma$. The values of $f_{\mathrm{abs}}\left(L_{\mathrm{b}}\right)$ in the individual bins of the histogram have been computed using the "observed", local, distribution of the 44 values of $\Sigma$ referred to in Sect. 4, and the bars represent the $68 \%$ confidence intervals calculated on the basis of the limited number of objects in that distribution. For the sake of neatness, in the following similar histograms, the bars will no longer be reproduced. For comparison, in the same figure $f_{\text {abs }}$ is also given for two choices of a "single" representative value of $\Sigma$, namely the median $\left(150 M_{\odot} \mathrm{pc}^{-2}\right)$ and the mean $\left(270 M_{\odot} \mathrm{pc}^{-2}\right)$ of the same distribution. In both cases, especially in the second, the prediction is significantly higher in all bins. This follows from the fact that (see Fig. 4), when a substantial fraction of objects span the interval from 0 to about $300 M_{\odot} \mathrm{pc}^{-2}$, the covering factor is extremely sensitive to the way the values of $\Sigma$ are distributed. In this respect, the prediction of $f_{\text {abs }}$ obtained adopting $\Sigma=270 M_{\odot} \mathrm{pc}^{-2}$ (as it can be judged from Fig. 4) is rather close to the ceiling attainable by pushing the distribution toward much higher values of $\Sigma$ than adopted here: a point on which we return in Sect. 7.

It is important to note that, without the "physical" limit of unity for $\lambda$, the result would be different. For instance, if no cut-off at $\lambda=1$ in Eq. (1) is imposed, the computation of $f_{\text {abs }}$ results is approximately a constant value, irrespective of luminosity. This occurs because a single mass range, $10^{7}-10^{7.3} M_{\odot}$, turns out to dominate at all luminosities, and the value of $f_{\text {abs }}$ to be close to the one pertinent to these mass values. Moreover, in this instance, we have no information to adopt from NT2007 in order to estimate the contribution of the masses below $10^{7} M_{\odot}$, a very serious cause of uncertainties, due to the relatively large number of SMBH in the mass range $10^{6}-10^{7} M_{\odot}$. Because the relationship between $\lambda_{\text {peak }}$ and $M_{\mathrm{BH}}$ remains qualitatively the same at the redshift values dealt with in the following section, the same type of result, with the same type of uncertainties, is also obtained at the other redshifts.

To illustrate the consequence of taking into account the downsizing effect mentioned in Sect. 3, we only need to change the leading assumption on $q$, namely adopt a proper dependence

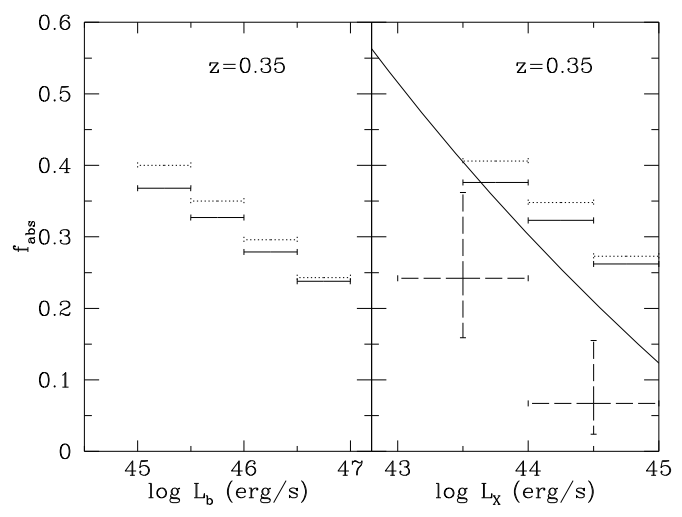

Fig. 7. Left panel: full line histogram, same as in Fig. 6; dotted line histogram, $q \propto M_{\mathrm{BH}}^{-1}$. Right panel: histograms as in left panel as a function of $L_{X}$; solid line from the global fit by LF2005; the data points, with Poissonian uncertainties, include the objects with $z=0.2-0.5$ in the sample used by LF2005.

of $q$ on $M_{\mathrm{BH}}$. The shape of the broad-line AGN mass function in the local universe, as obtained by Greene \& Ho (2007) above $10^{7} M_{\odot}$, suggests a dependence of the type $q \propto\left(M_{\mathrm{BH}} / 10^{7}\right)^{-0.5}$. To show that the knowledge of the exact difference between $F^{*}$ and $F$ is not very important for this range of mass, we assumed an even steeper correction as an exercise, namely $q \propto M_{\mathrm{BH}}^{-1}$. The result is shown in Fig. 7. The anticorrelation becomes somewhat steeper, but the difference overall is not particularly marked. This is so because, even though the absolute number of objects as a function of $L_{\mathrm{b}}$ has changed radically, the fraction of absorbed sources depends solely on the mix of values of $M_{\mathrm{BH}}$ contributing to each bin of luminosity. In particular, for instance, in the highest lumonosity bin the change is almost imperceptible because, despite the greatest change (decrease) in the absolute number of objects, the interval of $M_{\mathrm{BH}}$ values contributing to it have remained almost unchanged.

To proceed to a comparison with the results obtained in the hard X-rays, we need to turn from $L_{\mathrm{b}}$ to $L_{\mathrm{x}}$. The bolometric correction used comes from Marconi et al. (2004) and is not linear. We therefore recalculated Eq. (7) after converting $L_{\mathrm{b}}$ into $L_{\mathrm{x}}$. In the right panel of Fig. 7 the expectation for the two options on $q$ is compared with the results obtained by LF2005 around $z=0.35$. The two data points, with Poissonian error bars, represent the objects in their samples within the interval $z=0.2-0.5$, while the solid line represents the outcome at $z=0.35$ of their "global" fit of the intrinsic distribution after correcting for selection effects. It must be noted that the "global" fit given by LF2005 (dotted lines in their Fig. 11) also includes Comptonthick AGN with $10^{24}<N_{\mathrm{H}}<10^{25}$, so we recalculated their best-fit relation to take only the contribution of the Comptonthin AGN into account.

The difference between the data points and the line illustrates the relevance of the various selection effects that LF2005 take into account As it can be seen, our model predicts a decrease in the absorbed AGN fraction with increasing X-ray luminosity, but the slope of the predicted anticorrelation is not as steep as in LF2005.

\section{Redshift dependence in $\boldsymbol{f}_{\text {abs }}$}

We next move up to $z=0.7$, the highest redshift where the NT2007 findings do apply. The results on $f_{\text {abs }}$ (Eq. (7)), keeping $q=$ constant, are shown in the left panel of Fig. 8, as a function of $L_{\mathrm{b}}$, for $L_{\mathrm{b}} \geq 10^{45.4} \mathrm{erg} \mathrm{s}^{-1}$, where the completeness is ensured, 


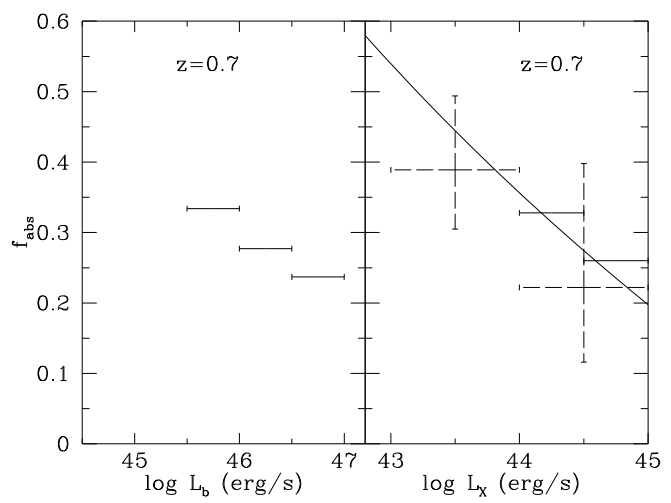

Fig. 8. Same as Fig. 7, but only with $q=$ const., at $z=0.7$. The data points include the objects with $z=0.55-0.85$ in the sample used by LF2005.

and in the right panel of Fig. 8, as a function of $L_{\mathrm{x}}$. Notably the values of $f_{\text {abs }}$ are almost identical to those calculated at $z=0.35$ for the same assumption on $q$. Despite the significant differences in $\lambda_{\text {peak }}\left(M_{\mathrm{BH}}\right)$ at the two redshifts, the parameters into play conjure to give essentially the same quantitative outcome: not surprisingly in the highest bin of $L_{\mathrm{x}}$, which remains dominated by the highest masses. Even if, at this particular redshift, the global best fit at $z=0.7$ from LF2005 (solid line) appears to be in reasonably good agreement with our prediction, the fundamental point is that the $z$-dependence of the $\lambda\left(M_{\mathrm{BH}}\right)$ distribution does not in itself introduce any increasing trend in $f_{\text {abs }}$, at least between $z=0.35$ and $z=0.7$. This conclusion is strengthened by the fact that a possible dependence of $q$ on $M_{\mathrm{BH}}$, reflecting changes in the shape of $F^{*}\left(M_{\mathrm{BH}}\right)$ above $10^{7} M_{\odot}$, is likely to be less steep at $z=0.7$ than assumed at $z=0.35$, for demonstrative purpose, in the previous section, and would therefore make no significant difference for the conclusion.

We now move to the local Universe. We used the information extracted from $\mathrm{H} 2004$, as described in Sect. 2 and summarised in Table 1. Figure 9, left panel, shows the results on $f_{\text {abs }}\left(L_{\mathrm{b}}\right)$ obtained in two ways, one directly from the curves in Fig. 3 of H2004, the other from their Lorentzian representation. The latter allowed us to go beyond $\log L_{\mathrm{b}}=46$, although one should be aware that the absolute number of very luminous objects is in any case very small in the local universe. Thus for our purpose, we consider uniportant that the H2004 sample lacks, by construction, the most luminous objects. In the interval $\log L_{\mathrm{b}}=44.8-46$, the $f_{\mathrm{abs}}$ expected from the $\mathrm{H} 2004$ curves or from their Lorentzian representation are practically indistinguishable.

After correcting $L_{\mathrm{b}}$ to $L_{\mathrm{X}}$, the expected $f_{\mathrm{abs}}$ is diplayed in Fig. 9, right panel. Here again the data points $(z \leq 0.2)$ and the best fit result from LF2005 are also given. The comparison shows that the predicted slope of the anticorrelation is definitely flatter than obtained by LF2005 at $z=0.1$.

To summarise these results, the $f_{\text {abs }}$ predicted as a function of $L_{\mathrm{b}}$ and $L_{\mathrm{X}}$, for the three values of $z$ covered (with $q=$ const. for $z=0.35$ and 0.7 ) are collected in Fig. 10. The simple conclusion that can be drawn immediately is that, despite the strong dependence of $\lambda$ on $M_{\mathrm{BH}}$ and on $z$, the normalization and slope of the anticorrelation between $f_{\mathrm{abs}}$ and $L_{\mathrm{X}}$, in the range of luminosities where they appear to change most quickly with $z$ according to LF2005, is expected to change very little, if at all.

We conclude this section by tentatively pushing our predictions beyond $z=1$. In this respect we note that, up to $z=0.7$ (see Sect. 2) the $\lambda-M_{\mathrm{BH}}$ relationships for the different values of

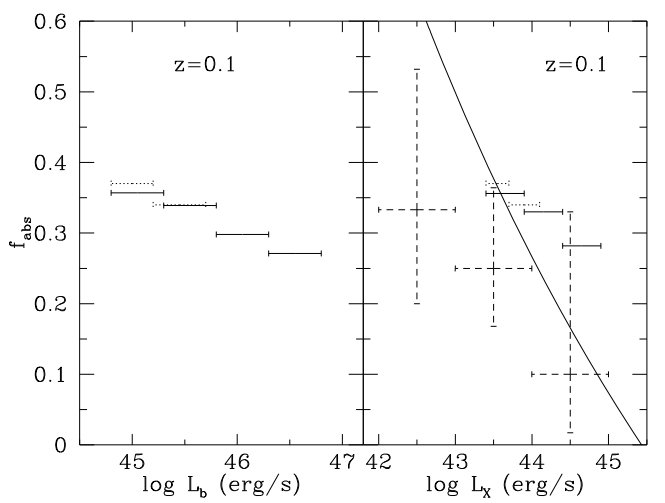

Fig. 9. Same as Fig. 7 at $z=0.1$, obtained using the $\mathrm{H} 2004$ results, see text. The dotted line histogram refer to the $\mathrm{H} 2004$ plots, the full line one to their representation with the function given by (1). The data points include the objects with $z \leq 0.2$ in the sample used by LF2005.

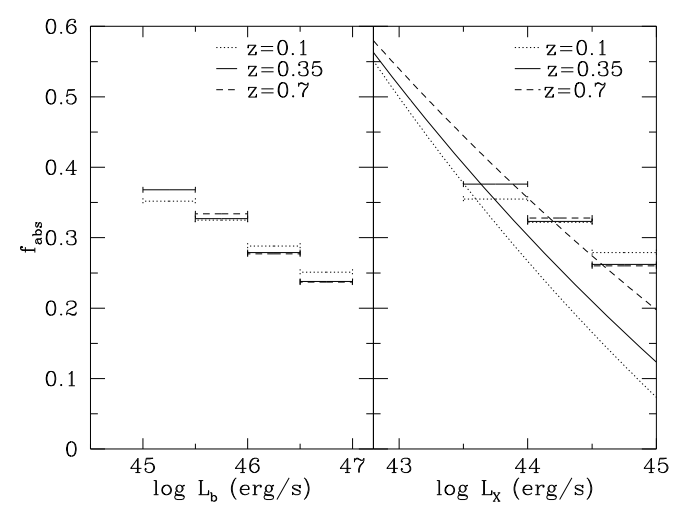

Fig. 10. The predicted $f_{\text {abs }}$ at $z=0.1$ and, with $q=$ const., at $z=0.35$, 0.7 . In the right panel the dotted, solid, and dashed lines are from the global fit by LF2005 at $z=0.1,0.35$, and $z=0.7$ respectively.

$M_{\mathrm{BH}}$ do converge. Thus we see no contradiction with results, in particular those obtained by Kollmeier et al. (2006) and mentioned in Sect. 1, going well beyond $z=1$, namely that out to $z$ about 4 the mean value of $\lambda$ seems to stabilize at a value, $\langle\lambda\rangle=0.25$ independent of both $M_{\mathrm{BH}}$ and $z$, and that its distribution is approximately lognormal. A recent investigation by Netzer et al. (2007) does essentially confirm those results for what concerns the mean value of $\lambda$ in the redshift bin 2.3-3.4 and the mass range $10^{8.8}-10^{10.7} M_{\odot}$, while the distribution is somewhat broader than found by Kollmeier et al. (2006). A simplified approach, which ignores the distibution and associates the same value of $\lambda$, equal to 0.25 , to any value of $M_{\mathrm{BH}} \geq 10^{7} M_{\odot}$, leads, when adopting the same $\Sigma$ distribution used previously, to the prediction of $f_{\text {abs }}$ given in Fig. 11, which, by construction, is independent of the SMBH mass function, and hence of $z$. The only, albeit small, difference relative to the prediction at $z=0.35$, shown for comparison in the same figure, is that the expected $f_{\text {abs }}$ becomes somewhat steeper, contrary to the LF2005 results.

\section{Discussion}

Despite the uncertainty on the actual form of $F^{*}\left(M_{\mathrm{BH}}, z\right)$ compared to that of the local $F\left(M_{\mathrm{BH}}\right)$, we have demonstrated that the predicted behaviour of $f_{\text {abs }}$ at high luminosities does not reproduce the increase with $z$ found by LF2005, as long as the distribution of $\Sigma$ is kept fixed and indifferent to the morphological type of the host galaxy. This result can be understood easily, 


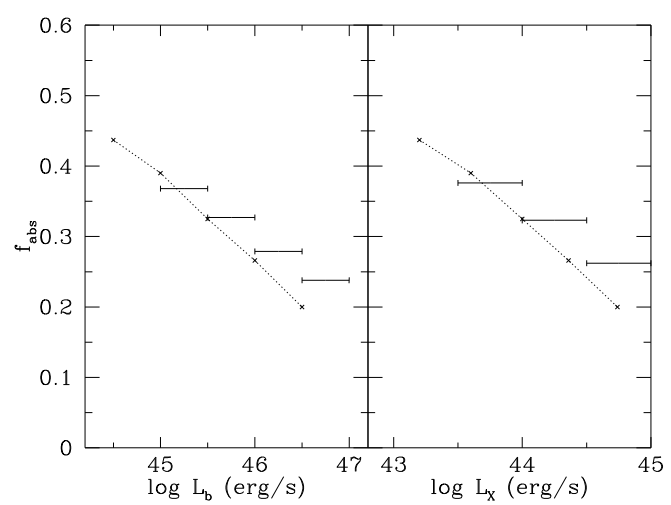

Fig. 11. The curve represent $f_{\text {abs }}$ at $z$ greater than 1 , predicted after adopting a fixed value of $\lambda=0.25$ irrespective of the SMBH mass. The histogram of the prediction at $z=0.35, q=$ const., is shown for comparison.

because the trend in $\lambda\left(M_{\mathrm{BH}}, z\right)$, in particular the way this quantity for different masses converge upwards with increasing $z$, implies that the higher the redshift, the higher the relative contribution of the more massive SMBH (with the lower $C$ ) at high luminosities. Another way to appreciate this point is to note that, being $L_{\mathrm{E}}\left(10^{8} M_{\odot}\right)=10^{46} \mathrm{erg} \mathrm{s}^{-1}\left(L_{\mathrm{X}}=10^{44.5} \mathrm{erg} \mathrm{s}^{-1}\right)$, beyond this luminosity only SMBH with higher masses contribute whose $C$ stays in the range about 0.3 ; therefore, an increase in $f_{\text {abs }}$ above this range cannot be predicted.

We are therefore left to operate on the other parameter of the model, namely $\Sigma$. On this quantity the empirical information we would need is practically missing, and only ad hoc assumptions can be made. It should be noted immediately that, as shown in Fig. 6, the increase in a "typical" value of $\Sigma$ shifts $f_{\text {abs }}$ upwards, without markedly altering its slope, in fact just rendering it somewhat steeper. Furthermore, in Sect. 5 we emphasised that the uppermost values in Fig. 6 practically represent a ceiling, as a consequence of the almost flat progression of the $C$ with incresing $\Sigma$ beyond about $300 M_{\odot} \mathrm{pc}^{-2}$, irrespective of $M_{\mathrm{BH}}$ (see Fig. 4). Thus, in the context of our model, there are limits on the values that $f_{\text {abs }}$ can achieve, no matter the assumptions we are willing to make.

As an exercise, let us assume that, in the local universe, the $\Sigma$ distribution adopted in the previous two sections applies only to the late type galaxies, the early types being comparatively, on average, much poorer in molecular gas (Sect. 4). For simplicity, we assume that $\Sigma$ is practically zero in the early type galaxies. Because the SMBH above $10^{7.5-8} M_{\odot}$ are mainly hosted by this type of galaxies, above $L_{X}=10^{43.5}$ this assumption implies a major drop in $f_{\mathrm{abs}}$, as illustrated in Fig. 12 at $z=0.1$, and the discrepancy with respect to the best fit by LF2005 is much stronger than in Fig. 9. Thence, to obtain a prediction closer in the local universe, as well as with increasing $z$, to the results in LF2005, one would need to assume that the presence of obscuring gas in at least the more massive early type galaxies (with the appropriate values of $\Sigma$ and organised as the model requires) is correlated with the nuclear activity and $\Sigma$ itself increases with $z$. One might adopt as a hint, in favour of the latter assumption, the increase with $z$ in the "volume-averaged" star formation rate (SFR) (see also $\mathrm{H} 2004$ for further considerations). However, this is not so straightforward, because the evolution of the X-ray luminosity function of AGN is not only in their luminosity but also in their number density: thus, what occurs in the unit volume does not necessarily apply to the individual objects. Perhaps the simplest speculation, that one can naively propose, is that the quantity $\Sigma$

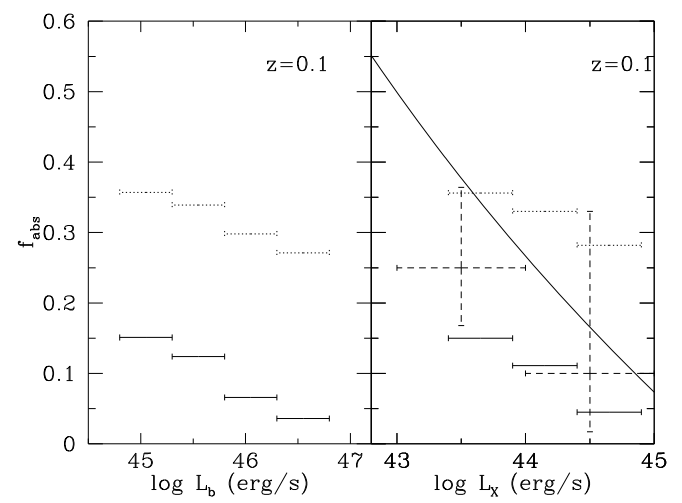

Fig. 12. Same as Fig. 9, with the addition of the $f_{\text {abs }}$ (full line histogram) predicted assuming $\Sigma=0$ in early type galaxies.

effectively present, or at least surviving during the activity phase, increases with $\lambda$. However, as noted above, it would remain hard for our model to explain values of $f_{\text {abs }}$ greater than about 0.3 at the highest luminosities.

Unfortunately, no theoretical picture of the gas spatial distribution and kinematics, the interplay with the SMBH growth, on the one hand, and the SFR on the other, has been developed so far to a degree of (physically consistent) detail necessary to sensitively adjust the prediction of $f_{\text {abs }}$. On the observational side, we lack sufficient data to proceed empirically, as we have done with $\lambda$.

We finally need to point out the non-negligible difference between the optical and the X-ray selection of AGN. Following Rigby et al. (2006) (see also Fiore et al. 2003; Cocchia et al. 2007, and references in all three papers), we stress that a substantial fraction of AGN selected through their X-ray emission and classified as such on the basis of their $L_{X}$ would be missed through an optical spectroscopic classification (this fraction increases from about $15 \%$ locally to about $50 \%$ at $z$ around 1). These objects could perhaps reduce the difference between our predictions and the $f_{\text {abs }}(z)$ found by LF2005. Such a possibility could be tested only after a properly complete investigation of the $\lambda$ of these objects is carried out.

\section{Conclusions}

In the light of recent new results on the behaviour of the Eddington ratio $\lambda$ as a function of the SMBH mass and of the redshift, we have revisited the predictions of the model presented in PapI. This model naturally predicts an anti-correlation between the fraction $f_{\text {abs }}$ of X-ray obscured, Compton-thin AGN, and $M_{\mathrm{BH}}$, provided there is interstellar molecular gas with appropriate values of surface density $\Sigma$ approximately within 25 to $450 \mathrm{pc}$ (depending on $M_{\mathrm{BH}}$ ) from the active nucleus. We used the newly found $\lambda\left(M_{\mathrm{BH}}, z\right)$ dependence ( $z$ from the local universe to 0.7 ) and a distribution of $\Sigma$, irrespective of the morphological type of the host galaxies and invariant with $z$, to convert that prediction into one versus $L_{\mathrm{X}}$. The results appear to reproduce, at least qualitatively, the anti-correlation between $f_{\mathrm{abs}}$ and $L_{\mathrm{X}}$ claimed by several authors, but not the still controversial claim of an increase in $f_{\text {abs }}$ with $z$. The latter increase, particularly in the form claimed by LF2005 on which we did concentrate, might possibly be reproduced by assuming that the typical values of $\Sigma$ do increase with the redshift and, furthermore, that in the early type galaxies, at least in the more massive ones, the presence of this quantity is correlated in time with the activity of their 
nucleus. At the highest luminosities, however, according to our model, $f_{\text {abs }}$ can hardly exceed 0.3 .

For the sake of completeness, it should be noted that the results just summarised are sensitive to the presence of a cut-off in the $\lambda\left(M_{\mathrm{BH}}, z\right)$ distribution, and they correspond to the case where the cut-off is placed at the physical limit $\lambda=1$.

Steps forward, that are relevant not only to the model discussed in this paper can be made at least in two ways. The first and most obvious one requires systematic investigation of the amount of molecular gas in an appropriately selected sample of early type galaxies, both active and inactive, out to redhifts of 0.5 at least. The line strength and profile may not tell us enough about the spatial distribution, but much about the amount and the kinematics of the gas. The former property needs to wait for observations with a large interferometric array of telescope, such as ALMA. The second step is somewhat indirect and therefore requires interpretation. The method is described and applied to a handful of broad-line objects in Maiolino et al. (2007). It allows an estimate of $C$ to be inferred for each object on the basis of measurements of infrared spectral features due to dust reradiation of the ultraviolet from the active nucleus. It is worth noting that Maiolino et al. (2007) find an anti-correlation between $C$ and either the luminosity or the mass, thus leaving completely open at present which of the two parameters is the one "physically" driving the anti-correlation. This sort of degeneracy could be resolved by comparing the covering factor of objects with similar $M_{\mathrm{BH}}$ and widely different values of $L_{\mathrm{b}}$, hence of $\lambda$. According to our model a similar value of $C$ should be found in such objects.

Acknowledgements. The authors are particularly grateful to Fabio La Franca for his help in assessing the figures with the X-ray data, and thank several colleagues for enlightening discussions. They acknowledge financial support from the ASI (grant 1/023/05/0) and MiUR (2006025203).

\section{References}

Akylas, A., Georgantopoulos, I., Georgakakis, A., Kitsionas, S., \& Hatziminaoglou, E. 2006, A\&A, 459, 693

Antonucci, R. R. J. 1993, ARA\&A, 31, 473

Barger, A. J., Cowie, L. L., Mushotzky, R. F., et al. 2005, AJ, 129, 578

Batcheldor, D., Axon, D., Merritt, D., et al. 2005, ApJS, 160, 76
Bettoni, D., Galletta, G., \& Garcia-Burillo, S. 2003, A\&A, 405, 5

Combes, F., Young, L. M., \& Bureau, M. 2007, MNRAS, 377, 1795

Cocchia, F., Fiore, F., Vignali, C., et al. 2007, A\&A, 466, 31

de Vaucouleurs, G., de Vaucouleurs, A., Corwin, H. G., et al. 1991, Third

Reference Catalogue of Bright Galaxies (RC3) (New York: Springer-Verlag)

Dong, X. Y., \& De Robertis, M. M. 2006, AJ, 131, 1236

Dwelly, T., \& Page, M. J. 2006, MNRAS, 372, 1755

Ferrarese, L., \& Ford, H. 2005, SSRv, 116, 523

Fiore, F., Brusa, F., Cocchia, F., et al. 2003, A\&A, 409, 79

Greene, J. E., \& Ho, L. C. 2007, ApJ, 667, 131

Hasinger, G. 2004, Nucl. Phys. B, 132, 86

Heckman, T., \& Kauffmann, G. 2006, New Astr. Rev., 50, 677

Heckman, T., Kauffmann, G., Brinchmann, J., et al. 2004, ApJ, 613, 109 (H2004)

Helfer, T. T., Thornley, M. D., Regan, M. W., et al. 2003, ApJS, 145, 259

Ho, L. C. 2002, ApJ, 564, 120

Hopkins, P. F., Richards, G. T., \& Hernquist, L. 2007, ApJ, 654, 731

Kaspi, S., Smith, P. S., Netzer, H., et al. 2000, ApJ, 533, 631

Kaspi, S., Maoz, D., Netzer, H., et al. 2005, ApJ, 629, 61

Kauffmann, G., Heckman, T. M., White, D. M., et al. 2003a, MNRAS, 341, 54

Kauffmann, G., Heckman, T. M., Tremonti, C., et al. 2003b, MNRAS, 346, 1055

Kollmeier, J. A., Onken, C. A., Kochanek, C. S., et al. 2006, ApJ, 648, 128

La Franca, F., Fiore, F., Comastri, A., et al. 2005, ApJ, 635, 864 (LF2005)

Lamastra, A., Perola, G. C., \& Matt, G. 2006a, A\&A, 449, 551 (PapI)

Lamastra, A., Matt, G., \& Perola, G. C. 2006b, A\&A, 460, 487

Maiolino, R., \& Rieke, G. H. 1995, ApJ, 454, 95

Maiolino, R., Shemmer, O., Imanishi, M., et al. 2007, A\&A, 468, 979

Marconi, A., Risaliti, G., Gilli, R., et al. 2004, MNRAS, 351, 169

Merloni, A., Heinz, S., \& di Matteo, T. 2003, MNRAS, 345, 1057

Netzer, H., \& Trakhtenbrot, B. 2007, ApJ, 654, 754 (NT2007)

Netzer, H., Mainieri, V., Rosati, P., \& Trakhtenbrot, B. 2006, A\&A, 453, 525

Netzer, H., Lira, P., Trakhtenbrot, B., Shemmer, O., \& Cury, I. 2007, ApJ, in press [arXiv: 0708.3787]

Pellegrini, S. 2005, ApJ, 624, 155

Perola, G. C., Puccetti, S., Fiore, F., et al. 2004, 421, 491

Richards, G. T., Strauss, M. A., Fan, X., et al. 2006, AJ, 131, 2766

Rigby, J. R., Rieke, G. H., Donley, J. L., Alonso-Herrero, A., \& Perez-Gonzales, P. G. 2006, ApJ, 645, 115

Sage, L. J., Welch, G. A., \& Young, L. M. 2007, ApJ, 657, 232

Shankar, F., Salucci, P., Granato, G. L., De Zotti, G., \& Danese, L. 2004, MNRAS, 354, 1020

Simpson, C. 2005, MNRAS, 360, 565

Treister, E., \& Urry, M. 2005, ApJ, 630, 115

Treister, E., \& Urry, M. 2006, ApJ, 652, L79

Tremaine, S., Gebhardt, K., Bender, R., et al. 2002, ApJ, 574, 740

Ueda, Y., Akiyama, M., Ohta, K., Miyaji, T. 2003, ApJ, 598, 886,

Welch, G. A., \& Sage, L. J. 2003, ApJ, 584, 260

Woo, J., \& Urry, C. M. 2002, ApJ, 579, 530

Young, L. M. 2002, ApJ, 124, 788

Young, L. M. 2005, ApJ, 634, 258 